\title{
The Optimal Experience: Social Identity and IT Identity as Antecedents of Group Flow in Social Media Use
}

\author{
Eric Curley \\ Kent State University \\ Ecurley1@kent.edu
}

\author{
Greta Polites \\ Kent State University \\ Gpolites@kent.edu
}

\begin{abstract}
Social media is a popular platform for daily communication and collaboration which supports interaction with online groups and communities. Prior research has investigated flow experiences in social media but only from an individual perspective. In this article, we examine group flow in the context of social media use. The key role played by the IT artifact, as well as the social nature of such use, require the addition of two new antecedents to group flow: IT identity and social identity. We propose that in conjunction with traditional flow experiences, group members' IT identity and social identification with the group will be strong predictors of group flow experiences. We further propose that group flow will lead to increased group exploration of the focal technology. Our research thus contributes to the growing literature on group flow by further developing its nomological network in social media usage contexts.
\end{abstract}

\section{Introduction}

In his seminal 1990 work, Csikszentmihalyi [1] described flow as a state of happiness where an individual takes control of what is happening in the moment and thereby creates the optimal experience. In his bestselling book, he positioned flow as a better way of experiencing life, informing readers how to enhance the quality of their experiences by setting goals that are positioned to match their skill level. Flow is a balance between challenge and skill while an individual is engaged in performing a task. Individuals can enter the state of flow when conditions are right, meaning that under the right circumstances flow becomes a possibility but not a guarantee. There are several identifying characteristics which are experienced while one is in the state of flow. These characteristics, such as an autotelic experience, make the activities surrounding flow inherently enjoyable, and thus contribute to the study of flow being important to a wide range of researchers. Since the release of Csikszentmihalyi's book, the scope of flow research has grown and expanded into many disciplines including information systems (IS), where it has been incorporated in research covering topics ranging from cognitive absorption and adoption to online gaming and social media usage [2][3][4][5].

Flow has become a powerful tool for researchers who are examining the reasons why people use technology. Flow is associated with many positive outcomes which is why it has seen increased attention over the past couple decades. For example, flow has been used to explain the adoption and continued use of social media platforms [5][6][7], online games [4], telepresence [8] and online use in general [9]. These studies indicate that positive flow experiences can encourage continued use of the technology for which flow is experienced. This has important implications for system designers who would like to improve their applications. Specifically, system developers are advised to pay more attention to the ways in which people use their systems, so that their design will encourage flow experiences if possible.

One major limitation of extant IS flow studies is that they have focused only on individual flow experiences while using these technologies. Recent work in other fields has begun to investigate flow that emerges from group interactions [10]. This new form of flow is known as group flow because it is formed through the interactions between the members of the group. Thus, the extant IS research incorporating flow lacks the scope of understanding that a multi-level approach provides.

Our study adds to the IS literature by looking at group flow to identify additional factors pertaining specifically to the nomological network of flow at the group level of analysis. This is particularly important in social media and group collaboration contexts where the focus of the platform is on facilitating interactions with other people. Collaboration technologies involve complex interactions between group members. This collaboration takes place in an environment where technology and social factors influence the communication between the group members. These factors have been studied on their own or in relation to other constructs but have not been studied together. Group flow is a part of collaborative social media that has been overlooked due to its early stages of 
development. Our study seeks to introduce the study of group flow into the IS literature by studying how IT identity, social identity and the individual flow experiences of the social media group members influence the formation of group flow and how group flow leads to the exploration of technology by the group. By incorporating group flow in the IS literature this research provides a foundation from which future researchers can conduct their own investigations.

Our research questions are as follows:

RQ1: Are IT identity, social identity and individual flow experiences associated with the experience of group flow?

RQ2: Does group flow lead to the exploration of technology features by the group as a whole?

We begin with a review of the literature on individual and group flow. Next, we present our research model and hypotheses. Then, we discuss our proposed methodology. We close with a discussion of implications and future research directions.

\section{Theoretical background}

\subsection{Flow theory}

The concept of flow as a state of optimal experience was first developed by Csikszentmihalyi [1]. He argues that when a person becomes completely involved in a task, (s)he enters into a state of flow - a positive experience that is accompanied by heightened enjoyment and productivity. The activities that create this flow state are autotelic, thus participation in these activities is sought after without need for a reward or other outcome [1].

Since flow was first introduced in 1990, it has been further refined by identifying dimensions representing different aspects of the flow experience which were found to be shared by the individuals studied [11]. The nine dimensions of flow as defined by Jackson \& Marsh [11] are: challenge-skill balance, action-awareness merging, clear goals, unambiguous feedback, concentration on the task at hand, sense of control, loss of self-consciousness, transformation of time, and autotelic experience. By establishing these dimensions, Jackson and Marsh were able to further develop instruments for measuring each dimension and thus measuring flow.

Chen et al [9] was one of the first IS studies to incorporate the concept of flow. Their research demonstrated that flow was experienced by Web users in reaction to the environment and content provided by the Web. They decomposed the nine dimensions of flow into the antecedents, experiences, and effects of flow as they pertain to the experience on the Web [9]. The antecedents which were identified were perceptions of clear goals, immediate feedback, and matched skills and challenges. These antecedents indicate that there is a combination of conditions created by the technology that encourages a flow experience. Clear goals and immediate feedback are possible given the nature of social media technology. Additionally, matched skills and challenges are a result of the capacity of the user to participate online. The technologies used in online activities today are particularly suited to accommodate these antecedents because of their inherent flexibility. For example, many online technologies offer real-time communication with the user, so that they are aware of their progress, a necessary part of the immediate feedback and clear goals [9].

\subsection{Group flow}

While there has been a vast array of research done to improve understanding of flow at the individual level of analysis, the concept of flow at other levels of analysis is still in the nascent stages of investigation. Nakamura and Csikszentmihalyi [12] summarized a handful of studies investigating flow experiences involving groups of individuals. These studies were published soon after the theoretical construct of flow was first conceptualized, but before researchers had begun to investigate differences between the levels of flow experience. Nakamura and Csikszentmihalyi argued that group flow is distinct from optimal individual experiences because the other members of one's group may or may not be in a state of flow [12]. These group experiences were termed shared flow, but their investigation of this new type of flow ended simply with a call for future research.

Sawyer [13] took this research a step further by considering flow as a property of the group, which lead to the study of collective experiences. Sawyer's research focused on musical collaboration, but his ideas on flow are transferable to any group where the members have an extrinsic collective goal(s) and the structures within the group to match the difficulty of the goal. These structures are what allows the group to communicate with each other and take on roles within the group. Sawyer argued that group flow is something that is more than just the work of the individual group members. Rather, the interaction between the members of the group is what forms group flow and impacts the group's performance.

More recently, Pels et al. [10] conducted a scoping review of the group flow literature. They further developed a generalized definition of group flow based 
on the definitions found in 18 separate publications. In the process of developing their definition, they noted that group flow has been represented by both individual and collective aspects. The individual aspects apply to how the individual experiences group flow whereas the collective aspects pertain to the features of the group itself [10]. These collective features of the group can be broken down into four types of features: (a) a specific shared state, (b) a specific group performance, (c) a specific group interaction, and (d) a specific social constellation comprised of a high social presence and positive relationships between the group members [10]. These features can be clearly seen in the concept of group flow developed by Sawyer [13], where musical groups enter a collective state during their performances and begin to interact at a higher level, which results in positive outcomes [10].

\subsection{Social identity}

Social identity focuses on one's relationship with others in social settings. Like other types of identity, social identity is a reflective process whereby an individual compares themselves to others in order to develop their self-concept. Tajfel et al. have outlined how interactions within the group are impacted by an individual's social identity [17]. Specifically, the interactions in the group are tailored to improve one's social identity or maintain an existing level of positive social identity. When the social identity of an individual falls too low and becomes negative, the individual will either leave the group or try to improve their self-concept in relation to that group.

We can formally define groups as social categories which the members of each group define by accepting some general norms. The members of the group will share a common definition of themselves and achieve a consensus about the evaluation of their group and membership [17]. These social groups then provide their members with a social identity which is used whenever interaction with the group occurs. Since a fundamental feature of group flow is intense member interaction, social interactions, as discussed in the literature on social identity, become an important component in group flow.

There are two main branches of social identity: relational identification and collective identification [18]. Both branches influence how people develop their social identity and what parts of the group matter more to the individual. The two forms of identity are distinct constructs which influence the social identification process [18]. In developing relational identity, the individual compares two groups, the ingroup and the out-group. The in-group is the group that the person belongs to. The out-group is another group which that individual views as relatively important. This process of comparison enables the individual to place these groups in a social structure where they perceive one group as being better or worse than the reference group [17]. This has become known as relational identification because the self-identity is based on the relationship one forms between themselves and others in the group as well as other groups. The other branch of social identity is collective identity, which is based on the properties of the group such as its positive qualities or the activities that the group performs [18]. The collective identity is based on the collective, not the individual, relationships. This leads the focus of the self-concept to be based on the goals of the group and the reputation of the group. [18]. Membership in the group is an important part of the self-identity.

\subsection{IT identity}

IT identity is a form of material identity [21] that is defined as "the extent to which an individual views use of an IT as integral to his or her sense of self" [22]. This definition reflects how technology has become embedded in our lives and a part of who we are. The hardware, software, and platform environment can all play roles in how a person relates to and embraces a particular social media service [22]. Further, the behavior of individuals in social contexts has been shown to depend on their IT identity [22]. Since social media is a technology driven environment for communication, it becomes necessary to consider IT identity when discussing group behavior on social media platforms.

The IT identity literature argues that we view ourselves as having an expanded self-concept in the presence of IT with which we identify, and without the resources made available by the IT, our self-perception shrinks [22]. Social media technologies enable us to behave in ways that otherwise would be impossible, such as the ability to instantly communicate with friends and family via a rich medium. The specific technology being used defines what these additional resources are, and the overlap between one's personal resources and technology enables the use of these technological resources [22].

IT identity is a fluid construct that changes very rapidly during the initial use of technology but slows in development once it has become part of one's self. This development phase is characterized by exploratory use of the technology [22][23]. This exploration slows as the technology and the features become part of the routine; however, the exploration process may resume if there is a new reason to process the interaction with the IT [22]. 
IT identity has been conceptualized as having three distinct but correlated dimensions: relatedness, emotional energy, and dependence [22]. Relatedness represents the connection that one has with a particular technology, such that people who possess high levels of relatedness will use the technology they identify with in more situations. Emotional energy represents positive feelings when working with the technology. People with higher emotional energy are likely to feel playful while using the technology, whereas people with low emotional energy are more likely to feel boredom at the prospect of using the technology. Finally, dependence represents one's reliance upon the technology. A person who identifies highly with a particular technology will feel dependent on it, and much of their social interaction will be organized around using it. Further, relationships with other people would be communicated through this technology, and the social environment would depend on its use.

\section{Research model}

Our research model (Figure 1) draws from the work of Sawyer [13], who argues that group flow is a property of the group as a collective unit, and that the emergence of group flow depends on the circumstances that the group is in. We extend the ideas that Sawyer initially proposed, and others have investigated, by integrating them with the identity literature, within a social media context. Several extant studies have investigated flow within the context of social media use, but these studies have been centered around the benefits of the individual experiences. Our study differs from prior work in investigating the predictors of group flow in social media use, and how group flow influences the group's behavior toward the use of technology. We propose a multilevel research model where the individual experiences of flow and two distinct types of identity are important factors that influence the formation and extent of group flow. We also propose exploratory use as an outcome of group flow.

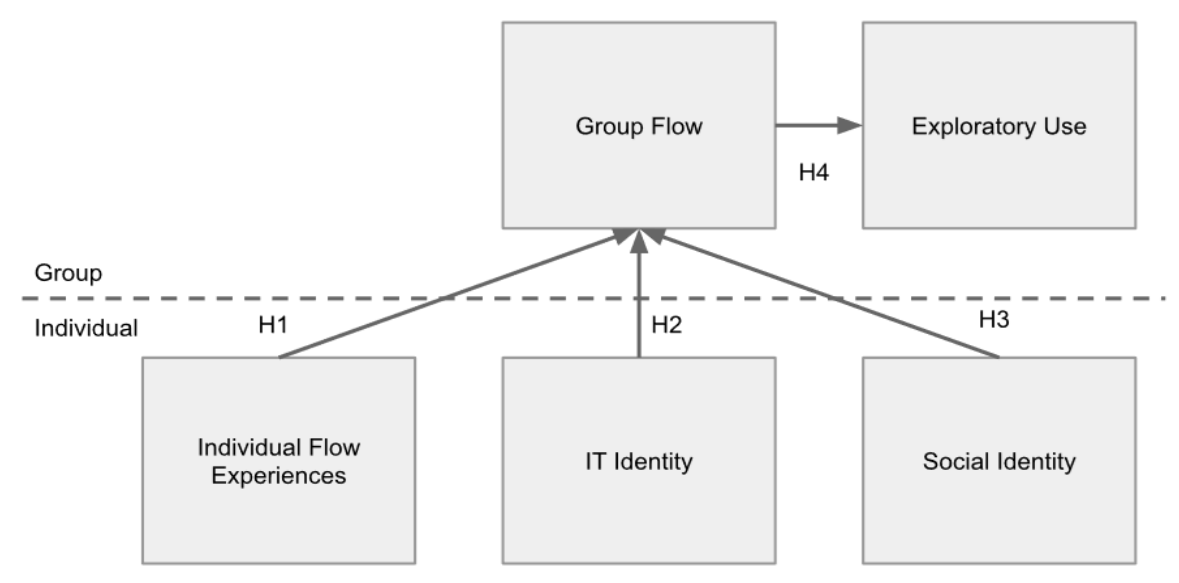

Figure 1. Research Model

\subsection{Antecedents of group flow}

3.1.1. Individual flow experiences. Individual flow experiences are associated with group flow experiences such that the group flow experience can be described as having two types of components: individual aspects and collective aspects [10]. Several studies have identified that the individual experiences of group flow have many of the same aspects as individual flow experiences [14][15][16]. As such, the individual flow experiences do not wholly account for the formation of group flow but they contribute to the individual aspects of group flow.
According to Pels et al., the main difference between group flow and the aggregation of individual flow experiences is a collective balance which exists in group flow experiences [10]. This balance could be based on the competency of the group, the group's state of mind, or the behavior of the group. Thus, the defining factor which makes the group balance different from that of the individual flow is that the balance in a group is shared amongst the members of the group. However, this does not discount the importance of the individual flow experiences in forming group flow. Heart \& Di Blasi, while describing an interactive team, state that "...[i]n these 
groups it is expected that all those involved are experiencing the nine characteristics of individual flow while concurrently engaging in a shared goal-oriented activity (. . .)." (p. 278) [14]. Zumeta et al. also indicate that flow characteristics are experienced in group flow settings such as the merging of action and awareness and the sense of control [16]. Both of these studies show that individual flow experiences are associated with group flow experiences and to a lesser extent that the antecedents of individual flow also influence the group members during group flow states. It is expected that under different social settings that the individual components of flow will vary in their importance when contributing to experience of flow [16]. Thus, we posit:

H1: As the flow experience of the individual group members increases, group flow will also increase.

3.1.2. Social identity. Both relational identity and collective identity are important to forming one's social identity. Relational identity is important to consider when investigating group flow because the relationships that an individual forms with members of the group would be considered part of the process that builds their relational identity. The relationship with group members allows for and improves the group flow experience. In a study on cooperative gaming, Kaye identified the key factors that determine the extent of group flow [19] as effective group communication, knowledge of others' skills and effective teamwork. A common aspect of these factors is that they all apply to the relationships one has with other members of the group as well as the goals of the group. This indicates that both aspects of social identity should impact group flow. Group communication and knowledge of others' skills are highly dependent on the relationships that one forms within the group, whereas effective teamwork is highly related to the group goals and tasks. A group such as a workgroup can be a setting for both types of identification to take place and for an individual to shape their identification [18]. Although considered distinct constructs, relational and collective identity have been shown to converge under circumstances of task interdependence and prototypicality [20].

Group flow is more likely when the individuals in the group have a social identity that aligns with that of the group. An individual may have a positive identity or negative identity associated with a group [17]. The positive identity associated with a group is largely formed from the favorable comparison with other reference groups. An unfavorable identity with a group will cause the individual to take actions to change that such as leaving a group. Not all situations involving group membership have an identity associated with them. For example, a member of a work group may be required to interact with customers and their participation in the group is not optional or an individual may have recently joined an online community which they have not already identified with. In these situations, the intense interactions required by group flow will be associated with individuals whose social identity is aligned with the group and will not be associated with individuals who do not align with the group or have yet to form an identification. Most social media interaction is expected to occur by choice. In that case the individual will have a social identity with the group they participate in. Those individuals who identify with the group more strongly are more likely to experience group flow. A favorable social identity will lead to prolonged interaction with the group, and further development of relationships with the group members. Thus, we posit:

H2: As the social identity of the individual group members increases, group flow will also increase.

3.2.3. IT identity. We posit that group flow is more likely when the individuals in the group strongly identify with the technology used for group communication and interaction. In the context of social media, the use of a specific technology such as a social media platform or chat app is fundamental to the online experience and the formation of group flow. This means that for that experience to be a positive one, the user needs to have a positive view of the technology that is being used or the experience may be disrupted by the use of the technology. The specific technology chosen by the group may not be one that the user identifies with. For example, an individual who joins a group who strictly uses Facebook may not have an IT identity with that platform and thus would experience using the platform differently than someone who identifies with Facebook. This may be due to the technology used by the platform or it may be other issues such as security or social issues which impact the user's IT identity with that technology. A person who does not use the primary platform of the group will find it more difficult to flow with the group compared to the other group members.

The dimensions of IT identity support the view that IT identity will be positively associated with group flow. The dimension of relatedness means that users of the technology are likely to use the technology in a wide variety of situations [22]. New situations may lead the user into group flow situations based on the technology they use. For example, an individual that relates with a social media platform such as Facebook may discover groups which have a Facebook presence and interact with those groups via that platform. Relatedness is an important part of understanding 
group flow because it influences the collective aspects of group flow in a social media context. Specifically, group interaction and a high social presence are affected by the relationship that the individuals have with the technology. Individuals who view themselves as being highly related to a social media technology will use it in their everyday life [22]. Everyday use of social media, especially with the group, will increase an individual's group interaction and social presence.

The second dimension of IT identity, emotional energy, should also affect group flow. Carter indicates that the emotional energy that is associated with an IT identity may have positive effects on other emotions associated with the use of that technology [22]. In contrast, low emotional energy may lead to boredom [22], which is an opposite state to flow. Since flow is considered the optimal experience and is associated with positive emotions, the level of emotional energy associated with IT identity will also impact how flow is experienced. High emotional energy will impact the shared state of the group and influence the positive relationships between group members. This is due in part to selective interaction where an individual chooses to surround themselves with others who reinforce their identity [22][24].

Finally, dependence on a technology is part of one's IT identity which impacts the group interaction and group performance. As Carter indicates, technology plays an important role in organizing our daily lives, communicating with friends and family, and understanding ourselves in our social environment. It is through the technological dependence that social media groups are able to exist and benefit their members. Thus, we posit:

H3: As the IT identity of the group members increases, group flow also increases.

\subsection{Consequences of group flow}

Due to the heightened enjoyment and overall positive experiences associated with group flow, we posit that groups experiencing flow when using collaboration and communication technologies such as social media platforms will be more inclined to explore new uses of these technologies. Few studies have looked at group flow in the context of technology use [19][25][15], but these studies did find that flow experiences in social contexts were associated with positive experiences and enjoyment. Further, studies of individual flow in e-commerce and instant messaging have shown flow to be directly associated with positive attitudes and exploratory behavior [26][27][28]. These studies indicate that since flow is a positive experience, one's interest in the activity is increased. The increased interest in the activity enables the individual to explore other areas of the technology to seek out further flow experiences. We expect that a similar effect takes place in groups where the group is exploring the technology. Thus, we posit that group flow will likewise lead the group to further exploration of the social media platform in use. This exploratory use could take many forms; for example, a group might begin using previously unutilized group management features provided by the platform, or it could begin using a newly released feature as part of the typical group discussion. The exploratory behavior of the group may drive exploration of features that go beyond the basic feature set used for interaction. Such as the use of screen sharing technology or video calls. Technology features such as these will be explored by the group as a result of the positive flow experiences. These new features can be viewed as enhancing the communication of the group by adding new mediums for the group to communicate. Since the exploration of additional features takes place as a group and is driven by the experiences of group flow, we posit:

H4: Higher group flow associated with a particular social media platform will lead to increased exploration of the platform as a group.

\section{Methodology}

\subsection{Pilot study}

We plan to first conduct a pilot study to assist in developing theoretically and empirically sound measures of group flow which are applicable in a social media context. Data collection for the pilot study will be based on an anonymous online survey where individuals will be asked to consider a specific example of past or current membership in an online group, such as a Facebook group or a WhatsApp chat group. The individuals to be surveyed will be asked to consider their experiences while interacting with the group of interest. They will then answer questions related to their individual flow experiences with the technology (outside of the group context), group flow perceptions, IT identity, and social identity. There are only a couple of restrictions that the participants in the survey must meet. First, they must currently be, or previously have been, a member of a social media group in which they actively participated. Second, the interaction between members of the group must be (or have been) primarily conducted online through a specific social media platform. 


\subsection{Measures}

The goal of the pilot study is to establish measurement scales which are appropriate for the research context. This goal is made necessary because the majority of flow research has been conducted in non-technology contexts [10]. The scales will be developed in multiple stages. Scales developed in previous research will need to be modified to account for the change in context and research agenda. Next, the new items will be reviewed by a small group of content area experts to ensure that the items are worded appropriately. Following any needed changes, we will conduct the pilot test. Participants in the pilot test will be given the ability to provide feedback on the survey in order to further refine the scales if necessary.

4.2.1 Individual and group flow. One of the biggest challenges in the study of group flow is how to properly measure the construct [10]. Pels et al.'s recent review indicates that there have only been twenty studies investigating group flow via some form of quantitative analysis. Of these twenty studies, only five studies developed a self-report scale to measure group flow based on measuring flow experiences and adapting the survey questions to a group perspective. One of these studies, Kaye [19], adapted the short version of the Flow State Scale [11], which is a popular method of measuring flow experiences in individuals, to measure both individual flow experiences and group flow perceptions while using technology. We will use the Flow State Scale with similar modifications as those made by Kaye, to measure group flow perceptions [19]. An example item to measure individual flow would be: "I was challenged, but I believed my skills would allow me to meet the challenge." A mirrored item to measure group flow would be: "The group seemed challenged, but I believed the group's skills would allow us to meet the challenge."

4.2.2 IT identity. IT identity will be assessed using the scale developed by Carter [29]. The wording of the items in her scale will be modified to reflect the social media domain. An example item would be: "Thinking about myself in relation to the social media platform my group uses, I am dependent on that social media platform."

4.2.3 Social identity. To measure social identity, we will be using the collective self-esteem scale developed by Luhtanen [30]. This scale was developed to measure the self-esteem and identity of an individual as it pertains to their group membership. This scale effectively measures the perception of one's social identity and self-esteem across four subdimensions: membership esteem, public collective self-esteem, private collective self-esteem, and identity. Since our model does not make any hypotheses about selfesteem, we have chosen to use only the identity subdimension to measure social identity. The items of this sub-scale will be adjusted to assess the social identity associated with the specific group referenced in the study. One of the studies conducted during the original development of the scale indicated that referencing a specific group in the scale did not compromise the effectiveness of the scale [30]. An example item would be: "The Facebook group I belong to is an important reflection of who I am."

4.2.4 Exploratory use. Our ultimate dependent variable represents the exploration of the focal technology by the group. In order to measure exploratory use, we will use a scale that was originally developed to measure individual exploration of new technology features [31]. Since exploratory use has been strictly investigated at an individual level in the past, we must adapt the extant items to fit a group perspective. An example item would be: "When our group was exploring the system, we tried to use a large range of new features that helped us interact with each other."

\subsection{Survey design}

After the pilot test has established the validity of the measures, we will test the research model via a survey of members of university student groups. The use of student groups is preferred over the use of an anonymous online survey, such as through Amazon Turk, because it allows access to all of the members of a given group for data collection purposes. This is important because in order to test the multi-level model involving group flow, we need data from members of the same group or groups. Trying to collect data from members of the same group using an online survey platform would be difficult due to the potentially large number of different group memberships present.

For this survey of student groups, members of each student organization will be asked about their involvement in their student organization. Since student organizations contain several members, we will be able to look at both the individual level and group level constructs using a multilevel modeling approach. The survey will ask about the social media platform that the group primarily uses for communication. By restricting the platform, the IT identity of the group members is expected to vary as everyone in the student organization is not expected to have the same identity with that technology. The survey will be conducted in two parts. The first part of the survey will ask about the antecedents and experience of group flow. The second 
part of the survey will be conducted approximately 3 months later and will ask about the exploratory use that has happened since the initial survey. As indicated by $\mathrm{Ke}$ et al. in the initial exploratory use development study, the use of a two-part survey solves some inherent problems associated with a standard crosssectional survey such as correlation between outcomes and precursors [31].

In addition to collection of information about the exploratory use, the survey will also ask about the group flow and individual flow of the members. This will allow for the comparison of the level of individual flow and group flow before and after that 3-month period. This will provide information about the spread of these constructs over the course of the 3-month period between surveys. The second survey doesn't look at the IT identity or the social identity because identity is not expected to change.

\subsection{Model testing}

The nature of our research model implies that there is a split level of analysis. Specifically, the group exists at the higher level of analysis, and the individual members of the group exist at the lower level of analysis. Multilevel models require specialized modeling techniques to test the effects across the levels of analysis. Multilevel modeling (sometimes referred to as hierarchical linear modeling) is a statistical technique which is used to analyze hierarchical data [32]. We propose to use a hierarchical linear modeling tool to test the research model, in order to properly account for the dependence between the individual scores (for individual flow experience, IT identity, and social identity) representing the lower level unit of analysis, and the scores for group flow representing the higher level unit of analysis, i.e., the group that the individuals are a member of. This method is preferred over other techniques such as aggregation of the data because of two reasons. The first is that the scores of the individuals are clustered into groups. The second reason is that the individual responses may not be independent [32].

\section{Limitations}

As with all research, our study comes with some limitations. The first limitation is the measures used. Under ideal circumstances, the investigation of group flow in social media would include both selfreported and objective measures. The use of objective measures for individual flow, IT identity, social identity, group flow, and exploratory in addition to self-reported measures would allow more precise measurement of these concepts. Study designs that accommodate the collective of objective measures could overcome the issues associated with self-report surveys. Second, even though the survey is collected in two parts it is still largely cross-sectional. This is because the antecedents of group flow and group flow are measured at the same point in time. This limits our ability to investigate the change in group flow experiences over time as individuals join, participate in, and leave a particular social media group. Lastly, the group diversity could influence the individual level perceptions, thinking, or behavior. Our study does not posit any relationships between types of group diversity and the individual level constructs, such relationships may exist but are outside the scope of our research. Future research should be directed toward investigating these potential relationships.

\section{Conclusions and future directions}

We argue that there is more to group flow than simply the individual flow experiences of the group's members. By incorporating IT identity and social identity as additional antecedents to group flow, and investigating one expected consequence of group flow, we take an important first step toward developing the nomological network surrounding group flow in a social media context. We do not discount the importance of individual level flow experiences in online environments, but rather we acknowledge that there are aspects of social media groups which contribute to a higher-level flow construct. The higherlevel construct of group flow combines aspects of individual experiences and identities in a way that is reliant on the experiences of the individuals and the group as a unit. This approach allows researchers and system designers to examine the positive experiences of both the individuals and the group. The positive experiences of the group in particular are expected to drive the group's future exploration of technology and use new features to prolong its positive experiences.

Our work offers a roadmap to researchers regarding new opportunities to incorporate group flow in the IS literature. We have undertaken the first steps to conceptualize the nomological network of group flow in a social media context. Future research could test the boundary conditions of this model and incorporate additional context-sensitive antecedents of group flow into the model. One such investigation might be directed at the individual components of flow and how certain group dynamics might alter the balance of those components. One component that might be of importance to researchers is the balance between challenge and skill. A group dynamic or technology might disrupt the balance between challenge and skill such that flow is more or less likely. 
Another such future research might be directed at the investigation of how group flow spreads within a group. Does it start with a few individuals who are flowing and other join in or does it emerge when a sufficiently large group all start flowing at once. Understanding the details of this process are outside the scope of this article but are interesting directions for future research.

In order to establish and test the proposed nomological network we have proposed new scales and techniques for measuring group flow that can be further tested and validated in other research contexts. In addition to investigating the antecedents of group flow in a social media context, we also propose that group flow will lead to positive group outcomes such as exploratory use of technology. The investigation of positive group effects such as exploratory use of technology is important for practitioners who seek to understand group dynamics in order to drive the development of their platforms. Features of the social media platform could be developed with the specific features of group flow experiences in mind, or the technological features could seek to target the antecedent factors in order to spur the increase in group flow experiences. Either way, the system developers should seek to improve the group flow experience because of the benefit that it has on the behavior of those involved. Future researchers can investigate other positive outcomes associated with flow experiences such as improved performance and heightened enjoyment as these may also be of interest for practical purposes.

\section{References}

[1] Csikszentmihalyi, M. (1990). Flow: The Psychology of Optimal Experience. New York: Harper and Row.

[2] Agarwal, R., \& Karahanna, E. (2000). Time flies when you're having fun: Cognitive absorption and beliefs about information technology usage. MIS quarterly, 665-694.

[3] Karahanna, E., Straub, D. W., \& Chervany, N. L. (1999). Information technology adoption across time: a crosssectional comparison of pre-adoption and post-adoption beliefs. MIS quarterly, 183-213.

[4] Choi, D. \& Kim, J. (2004). Why People Continue to Play Online Games: In Search of Critical Design Factors to Increase Customer Loyalty to Online Contents. Cyberpsychology \& Behavior, 7, 1. 11 - 24.

[5] Chang, Y. P. \& Zhu, D. (2012). The role of perceived social capital and flow experience in building users' continuance intention to social networking sites in China. Computers in Human Behavior, 28, 995-1001.
[6] Kaur, P. Dhir, A. Chen, S. \& Rajala, R. (2016). Flow in context: Development and validation of the flow experience instrument for social networking. Computers in Human Behavior, 59, 358-367.

[7] Shin, DH. \& Kim, W. (2008). Applying the Technology Acceptance Model and Flow Theory to Cyworld User Behavior: Implication of the Web2.0 User Acceptance. Cyberpsychology \& Behavior, 11.378-383.

[8] Pelet, J. Ettis, S. \& Cowart, K. (2016). Optimal Experience of Flow Enhanced by Telepresence: Evidence from Social Media Use. Information \& Management. 54, 1, 115-128.

[9] Chen, H., Wigand, R. T., \& Nilan, M. S. (1999). Optimal experience of web activities. Computers in human behavior, 15(5), 585-608.

[10] Pels, F., Kleinert, J., \& Mennigen, F. (2018). Group flow: A scoping review of definitions, theoretical approaches, measures and findings. PloS one, 13(12), e0210117.

[11] Jackson, S. A., \& Marsh, H. W. (1996). Development and validation of a scale to measure optimal experience: The Flow State Scale. Journal of sport and exercise psychology, $18(1), 17-35$.

[12] Nakamura J., Csikszentmihalyi M. (2014) The Concept of Flow. In: Flow and the Foundations of Positive Psychology. Springer, Dordrecht.

[13] Sawyer, R. K. (2003). Group creativity. Music, theater, collaboration. Psychology of Music 34, 2, 148 - 165.

[14] Hart, E., \& Di Blasi, Z. (2015). Combined flow in musical jam sessions: A pilot qualitative study. Psychology of Music, 43(2), 275-290.

[15] Kaye LK, Bryce J. Go with the flow. The experience and affective outcomes of solo versus social gameplay. Journal of Gaming \& Virtual Worlds. 2014; 6: 49-60.

[16] Zumeta, L., Basabe, N., Wlodarczyk, A., Bobowik, M., \& Páez, D. (2016). Shared flow and positive collective gatherings. anales de psicología, 32(3).

[17] Tajfel, H., Turner, J. C., Austin, W. G., \& Worchel, S. (1979). An integrative theory of intergroup conflict. Organizational identity: A reader, 56-65.

[18] Zhang, S., Chen, G., Chen, X. P., Liu, D., \& Johnson, M. D. (2014). Relational versus collective identification within workgroups: Conceptualization, measurement development, and nomological network building. Journal of Management, 40(6), 1700-1731.

[19] Kaye, L. (2016). Exploring flow experiences in cooperative digital gaming contexts. Computers in Human Behavior. 55, 286 - 29. 
[20] Sluss, D. M., \& Ashforth, B. E. (2008). How relational and organizational identification converge: Processes and conditions. Organization Science, 19(6), 807-823.

[21] Belk, R. W. (1988). Possessions and the extended self. Journal of consumer research, 15(2), 139-168.

[22] Carter, M. (2015). Me, my self, and I (T): conceptualizing information technology identity and its implications. MIS Quarterly, 39(4), 931-957.

[23] Bagayogo, F. F., Lapointe, L., \& Bassellier, G. (2014). Enhanced use of IT: A new perspective on post-adoption. Journal of the Association for Information Systems, 15(7), 3.

[24] Burke, P. J., \& Stets, J. E. (1999). Trust and commitment through self-verification. Social Psychology Quarterly, 62(4), 347-366.

[25] Kaye LK, Bryce J. Putting the "fun factor" into gaming. The influence of social contexts on experiences of playing videogames. International Journal of Internet Science. 2012; 7: 23-36.

[26] Hoffman, D. L., \& Novak, T. P. (2009). Flow online: lessons learned and future prospects. Journal of interactive marketing, 23(1), 23-34.
[27] Hoffman, D. L., \& Novak, T. P. (1996). Marketing in hypermedia computer-mediated environments: Conceptual foundations. Journal of marketing, 60(3), 50-68.

[28] Zaman, M., Anandarajan, M., \& Dai, Q. (2010). Experiencing flow with instant messaging and its facilitating role on creative behaviors. Computers in Human Behavior, 26(5), 1009-1018.

[29] Carter, M. (2013). IT Identity: Developing valid measures through CFA-based MTMM analysis. In International Conference on Information Systems (ICIS 2013): Reshaping Society Through Information Systems Design (Vol. 3, pp. 2398-2415).

[30] Luhtanen, R., \& Crocker, J. (1992). A collective selfesteem scale: Self-evaluation of one's social identity. Personality and social psychology bulletin, 18(3), 302-318.

[31] Ke, W., Tan, C. H., Sia, C. L., \& Wei, K. K. (2012). Inducing intrinsic motivation to explore the enterprise system: The supremacy of organizational levers. Journal of Management Information Systems, 29(3), 257-290.

[32] Kline, R. B. (2015). Principles and practice of structural equation modeling. Guilford publications. 